\title{
INTEGRATING DECISION MAKING CONDITIONS INTO DEA MODELS
}

\author{
Rokhsaneh Yousef Zehi ${ }^{1, *}$ And Adli Mustafa ${ }^{1}$
}

\begin{abstract}
Data Envelopment Analysis (DEA) is a popular non-parametric technique for the assessment of efficiency of a set of homogeneous decision making units (DMUs) with the same set of inputs and outputs. In the conventional DEA models, it is assumed that all variables are fully controllable. However, in the real-world applications of DEA, some of the variables are completely uncontrollable or partially controllable. In this paper, we are concerned about partially controllable variables which are called semi-discretionary variables. In DEA models, in the presence of semi-discretionary variables, decision makers have partial control on these variables and the proportional changes are possible to some extent. Previous DEA models with semi-discretionary variables consider a certain level of control on the variables which is fixed and it is given by decision makers or a higher authority. Since this level is usually given by experts, it is possible that in some cases all experts may not come up with an agreement, so in this paper we consider variable instead of fixed level of control on each semi-discretionary variable. In the presence of semi-discretionary variables, the proportional changes in inputs and outputs may not be feasible and as a result the obtained target value by conventional DEA models is not achievable for an inefficient DMU. Thus, we propose a bi-objective model to evaluate DMUs when modifying a variable to its target value should be managed by decision makers in a voting system. One of the advantages of the proposed model is including decision making conditions directly into a DEA model.
\end{abstract}

Mathematics Subject Classification. 90B50, 90C90, 90C05, 90C29.

Received December 24, 2019. Accepted May 1, 2021.

\section{INTRODUCTION}

Data envelopment analysis (DEA) pioneered by Farrel [9], who proposed a non-parametric frontier analysis for solving a linear programing to measure productive efficiency. Later the first DEA model which is called CCR model, was developed by Charnes et al. [7]. Since introducing the first DEA model, there has been a massive growth in the theory and application of DEA. From the theoretical aspect, various DEA models have been proposed in order to improve and extend DEA methodology and its applicability. In managerial applications, DEA has been widely used in different areas, such as health care, education, banking, agriculture, marketing, hospitality and many more.

DEA is a well-known technique for analyzing technical efficiency. By applying DEA model and representing the involved variables in the performance assessment correctly, more reliable efficiency scores will be achieved.

Keywords. Data envelopment analysis, decision making, efficiency, semi-discretionary variables.

1 School of Mathematical Sciences, Universiti Sains Malaysia, 11800 Pulau Pinang, Malaysia.

*Corresponding author: yousefzehi.rokhsaneh@gmail.com 
Also, DEA has been applied to identify technical inefficiencies of DMUs, and provide target value for each variable to improve inefficient DMUs [8]. In the conventional DEA models, whether it is radial or non-radial, it is supposed that all inputs and outputs are managed and under the control of a decision maker, in other words it is assumed that all variables are fully controllable which are called discretionary variables. However, in the real-world applications, there are some variables which are uncontrollable or partially controllable which are called non-discretionary and semi-discretionary variables, respectively.

DEA models in the presence of non-discretionary variables do not permit for any proportional changes for the non-discretionary variables. The first DEA model to account non-discretionary variables, was introduced by Banker and Morey $[2,3]$. They included the variables which increasing or decreasing their ratio is not the decision maker's authority in their proposed model. This model has been considered as the basic approach for the inclusion of non-discretionary variables in DEA models. In their study, non-discretionary variables do not have any direct effect on efficiency and they influence efficiency by constraint and it is due to removing the variable related to efficiency from the constraint related to the non- discretionary variables. Later, Ruggiero [16] introduced environmental non-discretionary variable and presented a modified DEA model to treat these variables correctly. In their study it has been claimed that the presented model by Banker and Morey tend to overestimate the technical inefficiency scores. In the presented model by Ruggiero, each unit only can be compared with those units which are faced with a same or worse environment in comparison with the under evaluation unit [16]. Camanho et al. [6] classified non-discretionary variables into two different types: Internal and external non-discretionary variables. Internal non-discretionary factors are those factors that can be considered as a part of the production process and therefore should be considered in the definition of the production possibilities set (PPS). On the other hand, External non-discretionary factors are those factors that affect the production process but cannot be considered as a part of it, and therefore they should not be allowed to define the PPS. Hence, they proposed a new model to assess the efficiency of DMUs with both internal and external non-discretionary variables while treating them differently in the model. Since introducing non-discretionary variables in DEA literature, this type of variables has been considered in a significant number of studies. Huguenin [12] developed a multi-criteria decisison making analaysis in order to select the most suitable nondiscretionary DEA model. Zadmirzaei et al. [20] analyzed the impact of external non-discretionary variables on the technical efficiency of forest management units. Zhang et al. [23] utilized a two-stage DEA model for resource allocation with the consideration of discretionary and non-discretionary inputs. Taleb et al. [18] proposed a two stage approach of super efficiency slack-based measure with non-discretionary variables and integer-valued data. Abdali and Fallahnejad [1] developed a bargaining game model to measure the efficiency of DMUs with twostage network structure in the presence of non-discretionary inputs. Khanmohammadi and Kazemimanesh [14] applied the context-dependent method for ranking DMUs with non-discretionary variables.

The studies that have been mentioned above considered the variables to be either discretionary or nondiscretionary. In the presence of semi-discretionary variables proportional changes are possible to some extent, so decision maker is able to control the changes in order to achieve possible and reliable target values which suits their organization's reality. Labor force is a good example of semi-discretionary variables, because it is possible to change it but not in any proportion. Consider a company that needs to lay off a high percentage of its employees in one occasion. However, by labor laws it might not be permitted, especially in public sector in which there are laws and regulations to adjust the number of employees. The managers have the authority to adjust the number of employees by a certain percentage and any percentage higher than that might be against the rules and regulations. From another aspect, even though if there are no regulations to reduce the number of employees in the short run, a high percentage of employee reduction affect employee's satisfaction which can severely affect company's efficiency in long term. Therefore, labor force can be considered as a semidiscretionary variable and the level of discretion can be suggested based on the regulations or the current status of the DMU. For example, in the result of an input oriented CCR model for an inefficient DMU it will be suggested to reduce the number of labor force (as an input) significantly which is not possible and operable, so these variables should be treated correctly in DEA models. Golany and Roll [10] were the first to take into account semi-discretionary variables in the efficiency assessment. They proposed an approach for handling 
semi-discretionary inputs and outputs which decision maker is able to change them by a certain percentage. In the proposed model an index is defined to specify the level of control or discretion for each input and output and their model aimed at reducing as much as possible the discretionary part of the inputs or outputs. Bi et al. [5] proposed a DEA model for two-stage production system with semi-discretionary variables in order to achieve more reliable improvement directions for inefficient DMUs. Later, Bi et al. [4] proposed a mixed integer linear model to handle semi-discretionary variables. This model despite considering the properties of semi-discretionary variables, also consider the relationship between non-discretionary inputs and the other inputs. Zerafat Angiz and Mustafa [21] provided a New aspect of representing semi-discretionary variables based on the fuzzy concept and the fuzzy interpretation of efficiency. They used the membership function to represent the semi-discretionary variable. Further Zerafat Angiz et al. [22] proposed a DEA model based on possibility set, which is equivalent to the traditional CCR model and they expanded that model to incorporate semi-discretionary variables into the model by defining the appropriate possibility set to represent semi-discretionary variables. Hu et al. [11] proposed a network DEA model based on the fuzzy concept and they extend the proposed model for the case with semi-discretionary variables.

In this paper we propose a bi-objective model to handle semi-discretionary variables which in, the level of discretion is not a fixed percentage and this level should be managed by decision makers in a voting system. For more clarification suppose we are evaluating banks, the number of employees can be one of the inputs. The manager of DMU has the authority to reduce the number of employees, but in some cases with a low efficiency score the suggested target value by DEA models is not acceptable, because reducing a high number of employees has some other side-effects such as service failure which can cause deleterious effect on DMU. The proposed model by Golany and Roll (for simplicity GR model in this paper) consider a certain level of control for this input which is a fixed percentage and it is given by decision maker. Since the decision maker in many organizations is not one person and the board of directors consists of a number of desicion makers, it is natural for them to provide different preferences and it is possible that in some cases they may not agree with a certain level of discretion. In this paper we consider variable instead of fixed level of discretion for each semi-discretionary variable in order to include the all suggested level of discretion in the model, therefore the model is able to choose the best level of discretion based on the technology. In fact, we include a decision making condition directly in a DEA model, while the other decision making methods, such as Analytic Hierarchy Process (AHP), firstly, they estimate the optimal weight for input and output, then secondly, use DEA to determine the optimal value for inputs and outputs $[13,15]$. Finally we apply the proposd model to 15 branches of a public company in Iran when the different decisions for the level of discretion are included and the level of discretion is chosen in the model and finally the efficiency score are provided. Also we show if we solve GR model by considering the obtained level of discretion from our proposed model, the results will be the same as our model.

The rest of this paper is organized as follows: section two provides a background of conventional DEA models, non-discretionary and semi-discretionary models. The new bi-objective model is given in section three. In section four, an example is given to illustrate the application of the proposed model. Finally, conclusion is presented.

\section{BACKGROUND}

\subsection{Data envelopment analysis}

Data envelopment analysis is a non-parametric method for evaluating relative efficiency of a set of homogeneous DMUs [8]. The reason for using relative term is that, the efficiency of each DMU under evaluation will be obtained by comparing it with all other DMUs in the technology. One of the basic radial DEA models is CCR model that was introduced by Charnes et al. [7]. For a clearer description of the CCR model, suppose, there are $n$ DMUs denoted by $j \in J$, which each of them produces a nonzero output vector $Y_{j}=\left(y_{1 j}, y_{2 j}, \ldots, y_{s j}\right)^{t} \geqslant 0$, using a nonzero input vector $X_{j}=\left(x_{1 j}, x_{2 j}, \ldots, x_{m j}\right)^{t} \geqslant 0$, where the superscript "t" indicates the transpose of a vector. Here, the symbol " $\geqslant$ " indicates that at least one component of $X_{j}$ or $Y_{j}$ is positive while the remaining inputs and outputs are non-negative. The CCR model based on these assumptions for evaluating the efficiency 
of $\mathrm{DMU}_{p}$, is formulated as the following mathematical programming:

$$
\begin{aligned}
& \min \theta_{p} \\
& \text { Subject to : } \\
& \sum_{j=1}^{n} \lambda_{j} x_{i j} \leqslant \theta_{p} x_{i p}, i=1, \ldots, m \\
& \sum_{j=1}^{n} \lambda_{j} y_{r j} \geqslant y_{r p}, \quad r=1, \ldots, s \\
& \lambda_{j} \geqslant 0, \quad j=1, \ldots, n
\end{aligned}
$$

where $\theta_{p}^{*}$ is the optimal value of the objective function and it is called the efficiency score of $\mathrm{DMU}_{p}$. $\mathrm{DMU}_{p}$ is efficient if and only if $\theta_{p}^{*}=1$ and $\mathrm{DMU}_{p}$ is inefficient, if and only if $0<\theta_{p}^{*}<1$.

\subsection{Non-discretionary DEA model}

The traditional CCR model is constructed based on the assumption that all the variables in the technology are fully controllable and the proportional changes for all variables are possible. However, in some real-world applications it may not be possible. Banker and Morey [2] were pioneers to address this issue explicitly in DEA. They divided inputs into two sets: discretionary inputs (D) and non-discretionary inputs (ND). The proposed input oriented model by them is given as follows:

$$
\begin{aligned}
& \min \theta_{p} \\
& \text { Subject to : } \\
& \sum_{j=1}^{n} \lambda_{j} x_{i j} \leqslant \theta_{p} x_{i p}, i \in D \\
& \sum_{j=1}^{n} \lambda_{j} x_{i j} \leqslant x_{i p}, \quad i \in N D \\
& \sum_{j=1}^{n} \lambda_{j} y_{r j} \geqslant y_{r p}, \quad r=1, \ldots, s \\
& \lambda_{j} \geqslant 0, \quad j=1, \ldots, n .
\end{aligned}
$$

The only difference between the constraint for discretionary and non-discretionary variable is ignoring factor $\theta_{p}$ from the right-hand side of the constraint related to non-discretionary inputs. In fact, the non-discretionary variables are considered as fixed values, which it is not possible to change them. Therefore, the input oriented model relies only on discretionary inputs and try to minimize the level of discretionary inputs to produce at least the same level of outputs.

\subsection{Semi-discretionary DEA model}

As previously mentioned, the non-discretionary variables are the factors which cannot be controlled by decision makers and decision makers do not have any authority to control or modify them. In other words, these variables are fixed for each DMU. Moreover, there are many real life application of DEA which the variables are not fully uncontrollable. For more clarification, consider a manager of company which has the right to authorize a limited amount of overtime but they have to follow the general guideline of their organization, so decision maker is able to control this factor to some extent.

It can be easily seen that in many of real life application of DEA, many of inputs or outputs have been treated as a non-discretionary variable while they should be treated as semi-discretionary variables. Golany and Roll [10] were the first to address these kinds of variables in a DEA model (for simplicity GR model in this paper). They defined $0 \leqslant \delta_{i} \leqslant 1$ and $0 \leqslant \delta_{r} \leqslant 1$ as a parameter to represent the degree of discretion that the DMU has on input $\mathrm{i}$ and output $\mathrm{r}$, respectively. $\delta=0$ (For any index) indicates that the factor is fully discretionary and $\delta=1$ means that the factor is fully non-dictionary. When a variable is a semi-discretionary it means that $\delta$ take a value between 0 and 1 . 
Golany and Roll introduced their DEA model in the presence of semi-discretionary variables as the following mathematical linear programming:

$$
\begin{array}{lr}
\operatorname{Min} \theta_{p}-\varepsilon\left[\sum_{r=1}^{s} s_{r}+\sum_{r=1}^{m} s_{i}\right] & \\
\text { Subject to : } & i=1, \ldots, m \\
\sum_{j=1}^{n} \lambda_{j} x_{i j}+s_{i}=x_{i p}\left[\theta_{p} . \delta_{i}+\left(1-\delta_{i}\right) \sum_{j=1}^{n} \lambda_{j}\right] & r=1, \ldots, s \\
\sum_{j=1}^{n} \lambda_{j} y_{r j}+s_{r}=y_{r p}\left[\delta_{r}+\left(1-\delta_{r}\right) \sum_{j=1}^{n} \lambda_{j}\right] & j=1, \ldots, n \\
\lambda_{j} \geqslant 0, & \forall i, r . \\
s_{i} s_{r} \geqslant 0 &
\end{array}
$$

It has been mentioned that by adding the convexity constraint associated with the BBC model (sum of $\lambda$ s equal to one) the parameter of degree of discretion will be disappeared from the constraint related to the outputs. Therefore, the BCC version of model (2.3) will not distinguish outputs according to their discretion level.

\section{HYBRID DEA DECISION MAKING MODEL}

In this section, we present the concept of decision making in a DEA model in an adjustment case, either workforce adjustment or equipment adjustment. In the conventional DEA models, the obtained result for an inefficient DMUs provides target values for variables in order to improve the efficiency of DMUs. However, in the presence of semi-discretionary variables, the proportional changes in inputs and outputs cannot find the proper target values in accordance with the reality of DMUs, because these changes may not be feasible and operable. For this reason, decision makers may want to control the changes in some variables to some extent while considering the current status of DMUs.

GR model as a DEA model to handle semi-discretionary variables, considers a fixed level of control for these variables which this level is given by decision makers. In some cases, decision makers may not agree with a certain level of control. Hence, it is preferable to include all suggested control levels and let the model choose the proper level. For more clarification consider the number of employees as input for an organization. However, this input can be reduced but not in any proportion compared to other inputs such as raw materials. The obtained result by conventional DEA models for an inefficient DMU suggest to reduce the number of their employees in order to promote the efficiency score. For a DMU with a low efficiency score it will be suggested to decrease a high number of its employees, while workforce lay off to the obtained target value by conventional DEA models will have some disadvantages to DMU, hence it is not desirable. Because laying off workforce will have other side-effects such as more workload to other workers, their dissatisfaction and etc. On the other hand, these disadvantages will have an effect on the efficiency of DMU in long term. Therefore, the decision maker is not able to reduce the number of employees to the target value and wants to have control on this factor to some extent. It means that the DMU is able to only scarifies a percentage of its workforce in order to promote its efficiency, and decision maker needs to know how the other inputs need to be changed. In a semi-discretionary model, it will be done by considering a certain level of control on the factor. When the members of directors or decision makers in an organization do not agree with a creation level of control, they use a voting-like system in order to find the most preferable level of control. It means that the decision makers suggest their preferred percentage of reduction for the considered variable which can be viewed as a level of control. Thus, corresponding to each suggested percentage we will have a suggested value. So, the level of control will be determined based on these suggested values and the optimal value which is equivalent with a level of control is the value which has 
the least distance to all these suggested values. In other words, we aggregate all suggested values in our model and let the model choose one of these values or a feasible value with a minimum distance from the all suggested values. It can be seen that the optimal value of reduction which is equivalent with a percentage of discretion for a semi-discretionary variable, can be used directly in the GR model and the result for efficiency scores will be the same. In other words, in the GR model the level of discretion is fixed while we want to expand a new model which we find the most appropriate level of discretion in a voting-like system.

In a voting-like system each expert will suggest that what percentage of input $\left(\forall i \in I_{1}\right)$ is controllable, which this percentage $\left(d_{i k}\right)$ is equivalent to a suggested value $s_{i k}$ (a percentage of the nominal value of input). Based on the frequency of the suggested percentage, the weight assigned to $s_{i k}$ will be obtained. The goal is to determine the controllable part of the input while we minimize its distance with all the suggested value by experts. In fact, by determining the controllable part of input, it is determined that what percentage of the initial value of input is controllable which this percentage can be directly applied in the GR model. Let $I_{1}$ be the set of semi-discretionary inputs and $\left(I \backslash I_{1}\right)$ is the set of other inputs. The efficiency of $\mathrm{DMU}_{p}$, when the level of discretion is not fixed and it will be managed in a voting-like system, can be determined by solving the following mathematical linear programming:

$$
\begin{array}{lc}
\operatorname{Min} \theta_{p} & \\
\operatorname{Min} \sum_{k=1}^{K} w_{i k}\left|\bar{x}_{i p}-s_{i k}\right| & \forall i \in I_{1} \\
\text { Subject to : } & \\
\sum_{j=1}^{n} \lambda_{j} x_{i j} \leqslant \bar{x}_{i p} . \theta_{p}+z_{i p}\left(\sum_{j=1}^{n} \lambda_{j}\right) & i \in I_{1} \\
\sum_{j=1}^{n} \lambda_{j} x_{i j} \leqslant \theta_{p} x_{i p}, & \\
\sum_{j=1}^{n} \lambda_{j} y_{r j} \geqslant y_{r p} & r \in\left(I \backslash I_{1}\right) \\
z_{i p}=x_{i p}-\bar{x}_{i p} & i \in I_{1} \\
\lambda_{j} \geqslant 0, & j=1, \ldots, n .,
\end{array}
$$

Model (3.1) is a multi-objective model, and it can be solved in a few stages. Symbol $w_{i k}$ denote the obtained weight (normalized frequency scores) for each suggested value, $\left(s_{i k} ; k=1, \ldots, K\right)$ represent the preferred values related to suggested percentages of reduction. $\bar{x}_{i p}$ is the controllable part of input and $z_{i p}$ is the uncontrollable part of input. The objective (Min $\sum_{k=1}^{K} w_{i k}\left|\bar{x}_{i p}-s_{i k}\right|$ ) is the decision making part of the model which determine the controllable part of semi discretionary inputs.

In the preferential framework, each decision maker suggests a possible percentage of reduction and the number of decision makers which suggest a particular percentage of reduction will be the frequency of that percentage of reduction. In addition, each percentage of reduction gives a value; $\left(s_{i k} ; k=1, \ldots, K\right)$. Let $d_{i 1}$ is the frequency of first suggested percentage of reduction for ith input and $d_{i K}$ is the frequency of $k$ th suggested percentage of reduction. $d_{i K}$ is the number of frequency for $s_{i k}$ which is the related value to $k$ th suggested percentage of reduction corresponding to $i$ th input. The normalized frequency number for $s_{i k}$ obtained as follows:

$$
w_{i k}=\frac{d_{i K}}{\sum_{k=1}^{K} d_{i k}} \quad i=1, \ldots, m .
$$


Assume, $\bar{x}_{i p}-s_{i k}=h_{i p k} \Rightarrow\left|\bar{x}_{i p}-s_{i k}\right|=\left|h_{i p k}\right|=h_{i p k}^{+}+h_{i p k}^{-}$; therefore by applying this substitution model (3.1) can be solved using the following multi-objective model.

$$
\begin{aligned}
& \operatorname{Min} \theta_{p} \\
& \operatorname{Min} \sum_{k=1}^{K} w_{i k}\left(h_{i p k}^{+}+h_{i p k}^{-}\right) \quad \forall i \in I_{1}
\end{aligned}
$$

Subject to :

$$
\begin{array}{ll}
\sum_{j=1}^{n} \lambda_{j} x_{i j} \leqslant \bar{x}_{i p} . \theta_{p}+z_{i p}\left(\sum_{j=1}^{n} \lambda_{j}\right) & i \in I_{1} \\
\sum_{j=1}^{n} \lambda_{j} x_{i j} \leqslant \theta_{p} x_{i p}, & i \in\left(I \backslash I_{1}\right) \\
\sum_{j=1}^{n} \lambda_{j} y_{r j} \geqslant y_{r p} & r=1, \ldots, s \\
\bar{x}_{i p}-s_{i k}-\left(h_{i p k}^{+}+h_{i p k}^{-}\right)=0 & \forall i, k, p \\
z_{i p}=x_{i p}-\bar{x}_{i p} & i \in I_{1} \\
\lambda_{j} \geqslant 0, & j=1, \ldots, n .
\end{array}
$$

Model (3.2) is a multi-objective model, therefore it can be solved by preemptive method $[17,19]$.

Although there are many other methods to solve multi-objective models, but we have used preemptive method, because in this preferential framework the most important objective for us is minimizing the distance between optimal controllable part of input value and the suggested values for controllable part of input. By applying this method, in the first stage, the objective function related to efficiency will be ignored, and the following model will be solved.

$$
\operatorname{Min} \sum_{k=1}^{K} w_{i k}\left(h_{i p k}^{+}+h_{i p k}^{-}\right) \quad \forall i \in I_{1}
$$

Subject to :

$$
\begin{array}{ll}
\sum_{j=1}^{n} \lambda_{j} x_{i j} \leqslant \bar{x}_{i p} . \theta_{p}+z_{i p}\left(\sum_{j=1}^{n} \lambda_{j}\right) & i \in I_{1} \\
\sum_{j=1}^{n} \lambda_{j} x_{i j} \leqslant \theta_{p} x_{i p}, & i \in\left(I \backslash I_{1}\right) \\
\sum_{j=1}^{n} \lambda_{j} y_{r j} \geqslant y_{r p} & \\
\bar{x}_{i p}-s_{i k}-\left(h_{i p k}^{+}+h_{i p k}^{-}\right)=0 & \forall i, k, p \\
z_{i p}=x_{i p}-\bar{x}_{i p} & i \in I_{1} \\
\lambda_{j} \geqslant 0, & j=1, \ldots, n .
\end{array}
$$

In the second stage, the following model should be solved in order to optimize the objective function related to efficiency. Besides, by adding an additional constraint we ensure that the previous objective is not degraded. If 
(L) is the optimal value for model (3.3), the additional constraint is as follows:

$$
\sum_{k=1}^{K} w_{i k}\left(h_{i p k}^{+}+h_{i p k}^{-}\right) \leqslant L .
$$

Then the model in second stage is given as the following mathematical linear programming.

$\operatorname{Min} \theta_{p}$

Subject to :

$$
\begin{array}{ll}
\sum_{k=1}^{K} w_{i k}\left(h_{i p k}^{+}+h_{i p k}^{-}\right) \leqslant L & \forall i \in I_{1} \\
\sum_{j=1}^{n} \lambda_{j} x_{i j}=\bar{x}_{i p} \cdot \theta_{p}+z_{i p}\left(\sum_{j=1}^{n} \lambda_{j}\right) & i \in I_{1} \\
\sum_{j=1}^{n} \lambda_{j} x_{i j} \leqslant \theta_{p} x_{i p}, & i \in\left(I \backslash I_{1}\right) \\
\sum_{j=1}^{n} \lambda_{j} y_{r j} \geqslant y_{r p} & \\
\bar{x}_{i p}-s_{i k}-\left(h_{i p k}^{+}+h_{i p k}^{-}\right)=0 & \forall i, k, p \\
z_{i p}=x_{i p}-\bar{x}_{i p} & i \in I_{1} \\
\lambda_{j} \geqslant 0, & j=1, \ldots, n \\
j=1,2, \ldots, n . &
\end{array}
$$

\subsection{Special case (target setting)}

In this section, we show that the proposed model in the previous section also can be used as a target setting model. DEA models can be used to assess how efficiently an organization utilize its resources to achieve the desired outputs. For an inefficient DMU, the conventional DEA models provides target values in order to achieve full efficiency score. In fact, in an input-oriented model, the obtained target value for an input show how that input should be minimized to promote the efficiency. In some cases, the obtained target value by conventional DEA models may not be operable due to the current status of DMU, so decision makers want to incorporate their preference for some factors. Consider the number of employees as an input, in this case we assume that the obtained target value for the number of employees (as an input) by conventional DEA models may not be operable due to the current position of DMU. Hence, the decision maker is not able to reduce the number of employees to the obtained target value and wants to fix a target value for the number of employees. For example, decision maker declare that they only can reduce 10 percent of their current number of employees. In this case, the inefficient DMU needs to minimize the other inputs and set a fixed target value for the number of employees to becomes efficient. When the fixed target value for some inputs or outputs should be managed in a voting-like system, we can aggregate all votes in the model and let the model to choose the target value for the specified inputs or outputs. Hence decision makers suggest their preferred percentage of reduction for the specified input or output and corresponding to each suggested percentage a suggested target value will be determined. So, the target value is the value which has the least distance to all these suggested target values.

Let $I_{1}$ the set of inputs that cannot be modified to their target value and the target value will be fixed for them and $\left(I \backslash I_{1}\right)$ is the set of other inputs. The efficiency of $\mathrm{DMU}_{p}$, when target values for some inputs are restricted to get the value which has the least distance from the suggested target values by decision makers, can 
be determined by solving the following mathematical linear programming.

$\operatorname{Min} \theta_{p}$

$\operatorname{Min} \sum_{k=1}^{K} w_{i k}\left|\bar{x}_{i p}-s_{i k}\right| \quad \forall i \in I_{1}$

Subject to :

$$
\begin{array}{ll}
\sum_{j=1}^{n} \lambda_{j} x_{i j} \leqslant \bar{x}_{i p} & i \in I_{1} \\
\sum_{j=1}^{n} \lambda_{j} x_{i j} \leqslant \theta_{p} x_{i p}, & i \in\left(I \backslash I_{1}\right) \\
\sum_{j=1}^{n} \lambda_{j} y_{r j} \geqslant y_{r p} & r=1, \ldots, s \\
\lambda_{j} \geqslant 0, & j=1, \ldots, n .
\end{array}
$$

In model (3.5) symbol $w_{i k}$ denote the obtained weight (normalized frequency scores) for each suggested target value, $\left(s_{i k} ; k=1, \ldots, K\right)$ represent the preferred target values related to suggested percentages of reduction and $\bar{x}_{i p}$ is the fixed target value for $x_{i p} ; \forall i \in I_{1}$.

Assume, $\bar{x}_{i p}-s_{i k}=h_{i p k} \Rightarrow\left|\bar{x}_{i p}-s_{i k}\right|=\left|h_{i p k}\right|=h_{i p k}^{+}+h_{i p k}^{-}$; therefore, by applying this substitution model (3.5) can be solved using the following model.

$$
\begin{array}{ll}
\operatorname{Min} \theta_{p} & \\
\operatorname{Min} \sum_{k=1}^{K} w_{i k}\left(h_{i p k}^{+}+h_{i p k}^{-}\right) & \forall i \in I_{1} \\
\text { Subject to : } & \\
\sum_{j=1}^{n} \lambda_{j} x_{i j} \leqslant \bar{x}_{i p} & i \in I_{1} \\
\sum_{j=1}^{n} \lambda_{j} x_{i j} \leqslant \theta_{p} x_{i p}, & \\
\sum_{j=1}^{n} \lambda_{j} y_{r j} \geqslant y_{r p} & \forall=1, \ldots, s) \\
\bar{x}_{i p}-s_{i k}-\left(h_{i p k}^{+}+h_{i p k}^{-}\right)=0 & j i, k, p \\
\lambda_{j} \geqslant 0, & j=1, \ldots, n .
\end{array}
$$

The preemptive method for solving a multi-objective model can be used to solve model (3.6). Again, the priority will be given to the objective that is aiming to minimize the distance between optimal target value and the suggested target values for inputs in set $I_{1}$. The following model in the first stage will be solved to determine the target value for inputs in set $I_{1}$. 
$\operatorname{Min} \sum_{k=1}^{K} w_{i k}\left(h_{i p k}^{+}+h_{i p k}^{-}\right) \quad \forall i \in I_{1}$

Subject to :

$$
\begin{array}{ll}
\sum_{j=1}^{n} \lambda_{j} x_{i j} \leqslant \bar{x}_{i p} & i \in I_{1} \\
\sum_{j=1}^{n} \lambda_{j} x_{i j} \leqslant \theta_{p} x_{i p}, & i \in\left(I \backslash I_{1}\right) \\
\sum_{j=1}^{n} \lambda_{j} y_{r j} \geqslant y_{r p} & r=1, \ldots, s \\
\bar{x}_{i p}-s_{i k}-\left(h_{i p k}^{+}+h_{i p k}^{-}\right)=0 & \forall i, k, p \\
\lambda_{j} \geqslant 0, & j=1, \ldots, n .
\end{array}
$$

In the second stage, the following model should be solved in order to optimize the objective function related to efficiency while considering fixed target for some inputs and the result from this model will show how the other inputs need to change in order to achieve full efficiency score.

$$
\begin{array}{ll}
\text { Min } \theta_{p} & \\
\text { Subject to : } & \forall i \in I_{1}(*) \\
\sum_{k=1}^{K} w_{i k}\left(h_{i p k}^{+}+h_{i p k}^{-}\right) \leqslant L & i \in I_{1} \\
\sum_{j=1}^{n} \lambda_{j} x_{i j} \leqslant \bar{x}_{i p} & \\
\sum_{j=1}^{n} \lambda_{j} x_{i j} \leqslant \theta_{p} x_{i p}, & \\
\sum_{j=1}^{n} \lambda_{j} y_{r j} \geqslant y_{r p} & \forall=1, \ldots, s) \\
\bar{x}_{i p}-s_{i k}-\left(h_{i p k}^{+}+h_{i p k}^{-}\right)=0 & \forall i, k, p \\
\lambda_{j} \geqslant 0, & j=1, \ldots, n .
\end{array}
$$

(L) is the optimal value for model (3.7), the additional constraint $\left(^{*}\right)$ is added to model to ensure that the previous objective is not degraded.

\section{NumERICAL EXAMPLE}

We show the application of the proposed model in Section 3 by applying it to the real-world data of 15 branches of a public company in Iran. The data, efficiency scores and target values by applying CCR model under constant return to scale are given in Table 1 . The input variables are the number of employees $\left(x_{1}\right)$ and budget $\left(x_{2}\right)$. The output variables are civil services $\left(y_{1}\right)$ and construction services $\left(y_{2}\right) .\left(x_{1}\right)$ is considered as a semi-discretionary variable and other variables are assumed to be discretionary.

The obtained result from Table 1 shows that for inefficient DMUs, the number of employees should be decreased in order to become efficient, however in some cases with a low efficiency score such as DMU 3, 7 and 12 , the suggested target value is not operable and decision makers are not able to reduce a high number of 
TABLE 1. Data, efficiency scores, target values.

\begin{tabular}{llllllll}
\hline \hline DMU & $x_{1}$ & $x_{2}$ & $y_{1}$ & $y_{2}$ & $\theta^{*}$ & $x_{1}^{*}$ & $x_{2}^{*}$ \\
\hline 1 & 520 & 2621 & 1631 & 844 & 0.977 & 508.20 & 2561.54 \\
2 & 568 & 2688 & 1729 & 742 & 0.860 & 476.75 & 2312.44 \\
3 & 681 & 3380 & 1146 & 408 & 0.451 & 306.84 & 1522.95 \\
4 & 529 & 2569 & 1960 & 821 & 1 & 529 & 2569 \\
5 & 364 & 1840 & 1028 & 543 & 0.893 & 325.14 & 1643.58 \\
6 & 831 & 4028 & 2140 & 973 & 0.746 & 619.86 & 3004.56 \\
7 & 743 & 3520 & 1351 & 630 & 0.550 & 401.08 & 1937.57 \\
8 & 487 & 2320 & 1209 & 789 & 1 & 487 & 2320 \\
9 & 392 & 2745 & 1686 & 897 & 1 & 392 & 2745 \\
10 & 465 & 3428 & 1567 & 690 & 0.784 & 364.33 & 2551.25 \\
11 & 966 & 4960 & 2526 & 997 & 0.691 & 667.84 & 3429.06 \\
12 & 582 & 2766 & 1456 & 640 & 0.718 & 410.10 & 1986.77 \\
13 & 672 & 3520 & 1427 & 864 & 0.731 & 491.30 & 2573.49 \\
14 & 1043 & 5821 & 2734 & 874 & 0.671 & 699.89 & 3906.11 \\
15 & 384 & 2160 & 1263 & 677 & 0.951 & 365.15 & 2053.97 \\
\hline
\end{tabular}

their employees. So they are willing to have control on this factor, but decision makers cannot come up with an agreement about the level of control. In order to cope with this problem, 50 members of board of directors have been asked to suggest their preferable possible percentage of reduction by considering the company's current status. Each possible percentage of reduction can be viewed as a level of control, for example if one of the decision makers declare that only 20 percent of the current number of employees is reducible, it means that the level of control for this factor is $0.2\left(\delta_{i}=0.2\right.$ in model $\left.(2.3)\right)$. The results of our proposed model are given in Table 2.

As we can see in DMU 3, 7 and 12 the efficiency score is low and the obtained target value for $x_{1}$ is not acceptable by decision makers and they want to define some limitation for them while trying to improve efficiency by achieving more applicable target values. For example, in DMU 3 the target value for $x_{1}$ is 306.84 , while the initial input value is 681 , it means laying off more than half of the employees which is not operable. The suggested level of control on $x_{1}$, by experts for DMU 3, 7 and 12, the frequencies (it means how many experts suggested a particular percentage of reduction) the result from model (3.1) and target values by model (3.1) are given in Table 2 .

The result from model (3.1) for DMU 3 shows the target value for $x_{1}$ is 555.36 which is more operable compare to the result from the conventional CCR model and the target value for $x_{2}$ is decreased to 1502.08. The chosen level of discretion for $x_{1}$ in DMU 3 by model (3.1) is $13 \%$ and if this level of discretion is directly applied in model (2.3), the same efficiency scores and target values will be obtained. In fact, model (3.1) is an expansion of model (2.3) when the level of discretion in not fixed and should be choose in the model. It is clear that model (2.3) is restricted to consider only one particular percentage of discretion, while the proposed model (model (3.1)) is more flexible in terms of level of discretion based on the particular conditions of the under assessing DMU. Another advantage of our presented model is in aggregating the suggestions by experts in our model to avoid infeasibility and impossible level of discretion. It may seem that model (3.1) always considers the level of discretion which has the highest frequency, but it is not correct. Model (3.1) based on the different conditions of DMUs uses the best level of discretion to find a feasible solution. For example, in DMU12, while $10 \%, 13 \%$ and $15 \%$ have higher frequency, but model (3.1) chooses the level of discretion equal to $12 \%$.

For the case of target setting for inefficient DMUs, decision makers are willing to incorporate their desire in order to improve the efficiency of such DMUs. For this case consider DMU 3 and 7 which in decision makers need to set a target for $x_{1}$ and such target values are not fixed and will be suggested by experts. The suggested target values for $x_{1}$ are incorporated in model (3.5) in order to determine the target value for the other inputs. 
TABLE 2. Suggested level of control on $x_{1}$ by 50 experts.

\begin{tabular}{|c|c|c|c|c|c|c|c|c|}
\hline $\mathrm{DMU}$ & $\begin{array}{l}\text { Suggested } \\
\text { level of } \\
\text { control }\end{array}$ & Frequency & $\begin{array}{l}\sum_{k=1}^{K} w_{i k}\left(h_{i p k}^{+}+h_{i p k}^{-}\right) \\
\text {in model }(3.1)\end{array}$ & $\begin{array}{l}\text { Chosen } \\
\text { level of } \\
\text { control by } \\
\text { model (3.1) }\end{array}$ & $\begin{array}{l}\theta^{*} \\
(\operatorname{model}(3.1))\end{array}$ & $x_{1}^{*}$ & $x_{2}^{*}$ & $\begin{array}{l}\theta^{*}(\text { model }(2.3)) \\
\text { With the same } \\
\text { level of control } \\
\text { chosen by } \\
\text { model }(3.1)\end{array}$ \\
\hline \multirow[t]{5}{*}{3} & $10 \%$ & 13 & 12.39 & $13 \%$ & 0.444 & 555.36 & 1502.08 & 0.444 \\
\hline & $12 \%$ & 11 & & & & & & \\
\hline & $13 \%$ & 16 & & & & & & \\
\hline & $15 \%$ & 3 & & & & & & \\
\hline & $18 \%$ & 7 & & & & & & \\
\hline \multirow[t]{4}{*}{7} & $10 \%$ & 14 & 49.04 & $25 \%$ & 0.550 & 636.26 & 1937.52 & 0.550 \\
\hline & $15 \%$ & 9 & & & & & & \\
\hline & $25 \%$ & 21 & & & & & & \\
\hline & $30 \%$ & 6 & & & & & & \\
\hline \multirow[t]{5}{*}{12} & $8 \%$ & 8 & 11.53360 & $12 \%$ & 0.718 & 520.88 & 1868.37 & 0.718 \\
\hline & $10 \%$ & 10 & & & & & & \\
\hline & $12 \%$ & 9 & & & & & & \\
\hline & $13 \%$ & 11 & & & & & & \\
\hline & $15 \%$ & 12 & & & & & & \\
\hline
\end{tabular}

TABLE 3. Target setting result for DMU 3 and 7.

\begin{tabular}{|c|c|c|c|c|c|c|}
\hline $\mathrm{DMU}$ & $\begin{array}{l}\text { Suggested } \\
\text { targets for } x_{1}\end{array}$ & Frequency & $\sum_{k=1}^{K} w_{i k}\left(h_{i p k}^{+}+h_{i p k}^{-}\right)$ & $\begin{array}{l}\text { Chosen tar- } \\
\text { get for } x_{1}\end{array}$ & $\theta^{*}(\operatorname{model}(3.5))$ & $x_{2}^{*}$ \\
\hline \multirow[t]{5}{*}{3} & 612.90 & 5 & 14.98 & 592.47 & 0.444 & 1502.08 \\
\hline & 599.28 & 12 & & & & \\
\hline & 592.47 & 11 & & & & \\
\hline & 578.85 & 9 & & & & \\
\hline & 558.42 & 13 & & & & \\
\hline \multirow[t]{5}{*}{7} & 676.13 & 15 & 16.35 & 668.70 & 0.550 & 1463.57 \\
\hline & 668.70 & 14 & & & & \\
\hline & 653.84 & 8 & & & & \\
\hline & 631.55 & 7 & & & & \\
\hline & 616.69 & 6 & & & & \\
\hline
\end{tabular}

In the voting system experts suggest that what percentage of the initial value of $x_{1}$ is preferred as the target value. The suggested target values, frequencies and the result form model (3.5) are given in Table 3.

As the result in Table 3 shows, for DMU 7, five different target values are suggested by experts and model (3.5) choose the target value for $x_{1}$ to be 668.70 with a frequency of 14 and by setting this target value for $x_{1}$ the target value for $x_{2}$ is 1463.57 .

\section{Conclusion}

In the conventional DEA models, it is assumed that all variables are fully controllable, however in many real-life applications there are some partially controllable (semi-discretionary) variables that cannot be fully controlled and decision makers are willing to have control on such variables in order to achieve more reliable target values. In some cases, decision makers cannot come up with an agreement that what level of a 
semi-discretionary variable is controllable and this level will be determined in a voting-like system. In this paper a multi-objective linear programming is proposed to include different level of discretion for semi-discretionary variables suggested by experts into a DEA model and let the model to choose the level of discretion which the proposed model ensures that the chosen level of discretion has the least distance from the suggested levels of discretion. If the chosen level of discretion by the proposed model (model (3.1)) is directly applied in the GR model, the same result will be obtained.

In addition, we proposed and adjustment of the model (3.1) that can be applied in a target setting case where decision makers need to include their desire and limitations on the target value for a variable, and decision makers cannot agree with particular value for the variable that its target value needs to be fixed. The suggested target values are included in the model and the target value will be chosen by model in a way that it has the least distance from all suggested target values. The advantage of the proposed model in this paper is that a decision making condition is directly included into a DEA model instead of applying another methods of decision making and applying the result to a DEA model. Also, in the GR model the level of discretion is fixed, while in the proposed model this level will be obtained in the procedure of solving the model. This paper provides the opportunity to study DEA models which may not only focus on the points on the efficient frontier. Proposing appropriate objective functions in order to target the other inner point of production possibility set can be an interesting topic for future researches.

\section{REFERENCES}

[1] E. Abdali and R. Fallahnejad, A bargaining game model for measuring efficiency of two-stage network DEA with nondiscretionary inputs. Int. J. Comput. Math. Comput. Syst. Theory 5 (2020) 48-59.

[2] R.D. Banker and R.C. Morey, The use of categorical variables in data envelopment analysis. Manage. Sci. 32 (1986) $1613-1627$.

[3] R.D. Banker and R.C. Morey, Efficiency analysis for exogenously fixed inputs and outputs. Oper. Res. 34 (1986) 513-521.

[4] G.B. Bi, J.J. Ding, Y. Luo and L. Liang, A new malmquist productivity index based on semi-discretionary variables with an application to commercial banks of China. Int. J. Inf. Technol. Decis. Mak. 10 (2011) 713-730.

[5] G.B. Bi, L. Liang and L.Y. Ling, DEA models for the chain-like systems with semi-discretionary variables. Syst. Eng. Electron. 29 (2007) 2052-2055.

[6] A.S. Camanho, M.C. Portela and C.B. Vaz, Efficiency analysis accounting for internal and external non-discretionary factors. Comput. Oper. Res. 36 (2009) 1591-1601.

[7] A. Charnes, W.W. Cooper and E. Rhodes, Measuring the efficiency of decision making units. Eur. J. Oper. Res. 2 (1978) 429-444.

[8] W.W. Cooper, L.M. Seiford and K. Tone, Introduction to data envelopment analysis and its uses: with DEA-solver software and references. Springer Science \& Business Media (2006).

[9] M.J. Farrell, The measurement of productive efficiency. J. R. Stat. Soc. Ser. A 120 (1957) 253-281.

[10] B. Golany and Y. Roll, Some extensions of techniques to handle non-discretionary factors in data envelopment analysis. J. Product. Anal. 4 (1993) 419-432.

[11] C.K. Hu, F.B. Liu, H.M. Chen and C.F. Hu, Network data envelopment analysis with fuzzy non-discretionary factors. J. Ind. Manag. Optim. 13 (2017) 0.

[12] J.M. Huguenin, Data Envelopment Analysis and non-discretionary inputs: How to select the most suitable model using multicriteria decision analysis. Expert Syst. Appl. 42 (2015) 2570-2581.

[13] B. Keskin and C.D. Köksal, A hybrid AHP/DEA-AR model for measuring and comparing the efficiency of airports. Int. J. Product. Perform. Manag. 68 (2019) 524-541.

[14] M. Khanmohammadi and M. Kazemimanesh, Ranking of efficiency in context-dependent data envelopment analysis with non-discretionary Data. Int. J. Ind. Math. 12 (2020) 197-207.

[15] S. Naghiha, R. Maddahi and A. Ebrahimnejad, An integrated AHP-DEA methodology for evaluation and ranking of production methods in industrial environments. Int. J. Ind. Syst. Eng. 31 (2019) 341-359.

[16] J. Ruggiero, On the measurement of technical efficiency in the public sector. Eur. J. Oper. Res. 90 (1996) 553-565.

[17] H.D. Sherali and A.L. Soyster, Preemptive and nonpreemptive multi-objective programming: relationship and counterexamples. J. Optim. Theory Appl. 39 (1983) 173-186.

[18] M. Taleb, R. Ramli and R. Khalid, Developing a two-stage approach of super efficiency slack-based measure in the presence of non-discretionary factors and mixed integer-valued data envelopment analysis. Expert Syst. Appl. 103 (2018) 14-24.

[19] Z. Yahia and A. Pradhan, Multi-objective preemptive optimization of residential load scheduling problem under price and CO2 signals. Proc. Int. Conf. Ind. Eng. Oper. Manag. (2019) 1926-1937.

[20] M. Zadmirzaei, S.M. Limaei, L. Olsson and A. Amirteimoori, Assessing the impact of the external non-discretionary factor on the performance of forest management units using DEA approach. J. For. Res. 22 (2017) 144-152. 
[21] M. Zerafat Angiz L and A. Mustafa, Fuzzy interpretation of efficiency in data envelopment analysis and its application in a non-discretionary model. Knowl.-Based Syst. 49 (2013) 145-151.

[22] M. Zerafat Angiz L, A. Mustafa, M. Ghadiri and A. Tajaddini, Relationship between efficiency in the traditional data envelopment analysis and possibility sets. Comput. Ind. Eng. 81 (2015) 140-146.

[23] J. Zhang, Q. Wu and Z. Zhou, A two-stage DEA model for resource allocation in industrial pollution treatment and its application in China. J. Clean. Prod. 228 (2019) 29-39. 